\title{
TRACKING CONTROL FOR PIECEWISE LINEAR SYSTEMS USING AN ERROR SPACE APPROACH: A CASE-STUDY IN SHEET CONTROL
}

\author{
B.H.M. Bukkems, W.P.M.H. Heemels, M.J.G. van de Molengraft, and M. Steinbuch
}

\begin{abstract}
This paper presents the design of tracking controllers for piecewise linear systems, with application to sheet control in a printer paper path. The approach that we will take is based upon an error space approach, which is derived from linear systems theory. We will show that due to the discontinuity in the piecewise linear system, the resulting model in error space consists of both flow conditions, describing the dynamics in each regime, and jump conditions, describing the error dynamics at the switching boundaries. Two types of controllers are proposed that result in either full or partial linearization of the closed-loop error dynamics. To show the effectiveness of the control design approach in practice, the sheet controllers are implemented on an experimental paper path setup.
\end{abstract}

\section{INTRODUCTION}

Control design and analysis of piecewise linear (PWL) systems has been given much attention in literature lately, see for example [1] for an overview. Most of the work, e.g. [2], [3], [4], [5] focuses on the stabilization of the system dynamics, meaning that regulation problems are considered, whereas tracking control problems have been given less attention in literature, as recognized by [6]. Also in [7], regulation problems are considered. An $H_{\infty}$ controller synthesis method for PWL systems based on a piecewise smooth Lyapunov function is presented, resulting in a stabilizing controller that guarantees disturbance attenuation up to a prescribed level. For the particular case of sheet tracking control, in [8] the design of $H_{\infty}$ output feedback controllers for PWL systems has been considered, with the goal to realize accurate tracking behavior of sheets in a printer paper path. Similarly to [8], also this paper considers the design of tracking controllers for PWL systems. However, in this case the controller design is based on an error space approach yielding state feedback controllers. It combines the analysis and synthesis techniques presented in [2], [3], [4], [5], i.e. the use of LMIs for the calculation of the controller gains and the use of Lyapunov theory to prove the stability of the controlled system, with state feedback approaches in error space, which are well-known for linear

This work has been carried out as part of the Boderc project under the responsibility of the Embedded Systems Institute. This project is partially supported by the Netherlands Ministry of Economic Affairs under the Senter TS program.

Björn Bukkems is with TMC Mechatronics, P.O. Box 700, 5600 AS Eindhoven, The Netherlands. bjorn. bukkems @ tme.n I

Maurice Heemels, René van de Molengraft, and Maarten Steinbuch are with the department of Mechanical Engineering, Dynamics and Control Technology, Technische Universiteit Eindhoven, P.O. Box 513, 5600 MB Eindhoven, The Netherlands. $\{\mathrm{w} \cdot \mathrm{p} . \mathrm{m} . \mathrm{h}$. heemels, m.j.g.v.d.molengraft, m.steinbuch\}@tue.nl systems [9]. By working in the error space, stabilization of the error dynamics is directly linked to tracking performance. In contrast with the formulation of linear models in the error domain, the formulation of the PWL sheet flow model in error space yields a discontinuous PWL model of the error dynamics. More specifically, the resulting model consists of both flow conditions, describing the dynamics in each regime, and jump conditions, describing the error dynamics at the switching boundaries. This gives rise to some complications in the controller design, as we will show. Based on the resulting model in error space, two types of stabilizing state feedback control laws are proposed. To show the effectiveness of the controller design technique, both simulation and experimental results will be presented.

As in [8], the case-study that is considered in this paper originates from the field of sheet handling in cut sheet printer paper paths. A schematic representation of such a paper path is shown in Fig. 1. Sheets enter the paper path at the Paper Input Module (PIM) and are transported to the Image Transfer Station (ITS) where the image is printed onto the sheet at high pressure and high temperature. After leaving the ITS, sheets can either re-enter the first part of the paper path for back side printing via a so-called duplex loop, or they can go to the finisher (FIN), where they are collected. The sheets are driven by pinches, which are sets of rollers that are, either individually or grouped together in sections, driven by motors. When we consider the sheet flow through the paper path, it can be seen that the driving motor-pinch combination changes as a function of the sheet position. Hence, the sheet flow through the paper path can

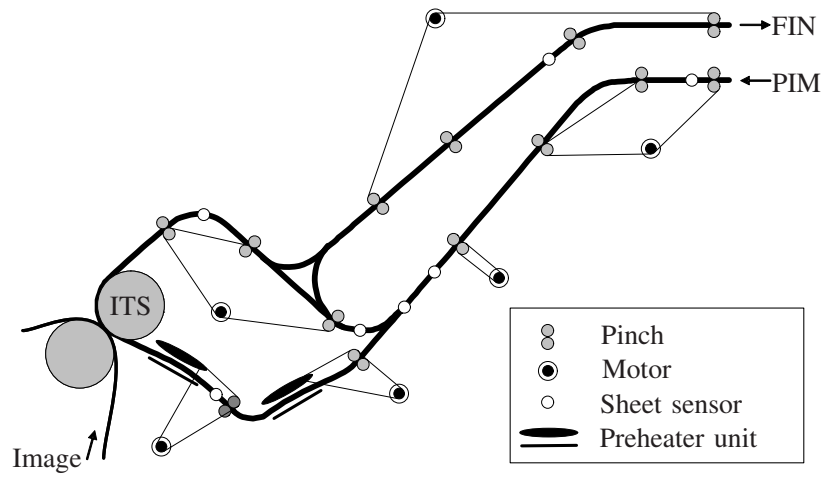

Fig. 1. Schematic representation of a printer paper path. 
be modeled as a PWL system. When we assume the sheet position to be known at all times, for example by measuring or reconstructing it, continuous feedback of this position information can be applied to achieve a prespecified desired sheet tracking performance [10], [11], [8], and therefore also the desired printing quality.

The remainder of this paper is organized as follows: in Section II, the system under consideration will be discussed in more detail, the control goal will be given, and the sheet flow model will be presented. In Section III, the controller synthesis techniques will be discussed, whereas in Section IV the experimental setup, that is used to validate the proposed control design will be discussed. The results of these experiments, together with the obtained simulation results, are given in Section $\mathrm{V}$, which at the end are followed by conclusions and recommendations.

\section{THE PAPER PATH CASE-STUDY}

In this paper, the focus will be on sheet feedback control design in a basic paper path. By considering this basic version, the essence of the control problem becomes clear. As a result, the switching nature of the system, caused by the consecutive changing of the driving pinch, naturally arises in the control design. The paper path is depicted in Fig. 2. It consists of three pinches (P1, P2, and P3), each of which is driven by a separate motor (M1, M2, and M3, respectively). The locations of the three pinches in the paper path are represented by $x_{P 1}, x_{P 2}$, and $x_{P 3}$, respectively. These locations are chosen such that the distance between two pinches is equal to the sheet length $L_{s}$, so the sheet can only be in one pinch at the same time. The sheet position, defined as $x_{s}$, is assumed to be known.

The control layout we adopt for the sheet feedback control design is the hierarchical (cascade) control structure presented in [8]. This control layout consists of low level (inner) motor control loops and a high level (outer) sheet control loop for tackling disturbances and uncertainties at the motor level and at the sheet level, respectively. In this paper, we assume the motor control loops to behave ideally, as a result of which the focus of the control design is on the design of the sheet control loop only. Consequently, the control goal we adopt for the basic paper path case study is the design of sheet feedback controllers that track a prespecified desired reference trajectory $x_{s, r}$. In this paper, this trajectory is chosen to be a first order reference profile, i.e. $\ddot{x}_{s, r}=0$, to focus on the essence of the resulting control design problem.

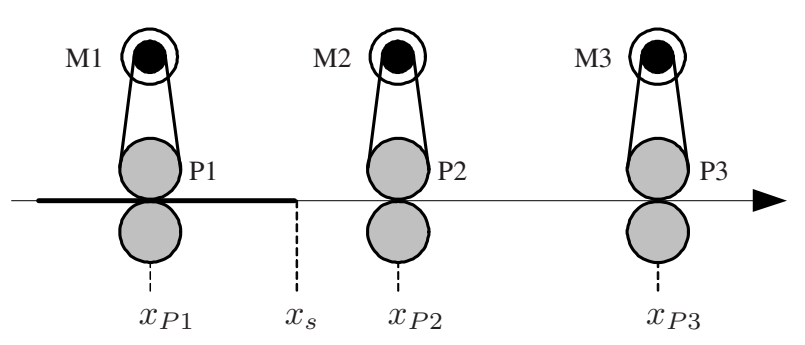

Fig. 2. Schematic representation of the basic printer paper path.
In the derivation of the sheet flow model for the basic paper path case-study, we take into account the fact that at each time instant the sheet is only driven by one motorpinch combination. Hence, only one of the motor velocities acts as an input for the sheet motion. As the sheet moves through the paper path, this input will change when the sheet arrives at the next pinch. This switching behavior can be easily captured in the PWL modeling formalism [12]. In the resulting sheet flow model, the mass of the sheet is not taken into account, as the effect of this mass is negligible with respect to the effect of the inertia of the motor-pinch combinations. Since flexibilities of the gear belts and slip between the sheet and the pinches are also not taken into account, the sheet velocity can be derived from the motor velocities via straightforward holonomic kinematic constraint relations that describe the relation between motor velocity and pinch velocity, and pinch velocity and sheet velocity, respectively. The sheet position is obtained by integrating the sheet velocity. The sheet flow model therefore consists of a switching integrator that can be represented as

$$
\dot{x}_{s}=B_{i} \underline{u} \quad \text { for } \quad x_{s} \in \mathcal{X}_{i}, i \in \mathcal{I},
$$

with the input matrices $B_{i}$ defined as $B_{1}=$ $\left[\begin{array}{lll}n_{1} r_{P 1} & 0 & 0\end{array}\right], \quad B_{2}=\left[\begin{array}{lll}0 & n_{2} r_{P 2} & 0\end{array}\right]$, and $B_{3}=\left[\begin{array}{lll}0 & 0 & n_{3} r_{P 3}\end{array}\right]$, respectively. In these definitions, $n_{i}$ represents the transmission ratio between motor $\mathbf{M} i$ and pinch $\mathrm{P} i$ and $r_{P i}$ represents the radius of the driven roller of pinch $i$. Furthermore, $\underline{u}$ is the column with inputs of the sheet flow dynamics: $\underline{u}=\left[\begin{array}{lll}\omega_{M 1} & \omega_{M 2} & \omega_{M 3}\end{array}\right]^{T}$, with $\omega_{M i}$ the motor velocities. The partitioning of the state space into the three regions is represented by $\left\{\mathcal{X}_{i}\right\}_{i \in \mathcal{I}} \subseteq \mathbb{R}$. Here, $\mathcal{X}_{1}=\left\{x_{s} \mid x_{s} \in\left[x_{P 1}, x_{P 2}\right)\right\}, \mathcal{X}_{2}=\left\{x_{s} \mid x_{s} \in\left[x_{P 2}, x_{P 3}\right)\right\}$, and $\mathcal{X}_{3}=\left\{x_{s} \mid x_{s} \in\left[x_{P 3}, x_{P 3}+L_{s}\right)\right\}$, since $x_{P 2}-x_{P 1}=L_{s}$ and $x_{P 3}-x_{P 2}=L_{s}$.

Given the sheet flow model (1) and the definition of the sheet tracking error, $e_{s}=x_{s, r}-x_{s}$, with $x_{s, r}$ the sheet reference position, the sheet flow model in error space can be derived. As a first step, the expression for the time derivative of the tracking error is considered:

$$
\begin{aligned}
\dot{e}_{s} & =\dot{x}_{s, r}-\dot{x}_{s} \\
& =\dot{x}_{s, r}-B_{i} \underline{u} \text { for } \quad x_{s, r}-e_{s} \in \mathcal{X}_{i}, i \in \mathcal{I} .
\end{aligned}
$$

Due to the switching character of the system, the right hand side of (2) can be discontinuous. Therefore the model of the sheet error dynamics will consist of two parts: the flow conditions that hold for the error dynamics of the various subsystems and the jump conditions that describe the relation of both $e_{s}$ and $\dot{e}_{s}$ just before and just after the switching moment.

For the derivation of the flow conditions, we differentiate (2) one more time to eliminate explicit dependencies on $x_{s, r}$ and its time derivatives, yielding

$$
\ddot{e}_{s}=-B_{i} \underline{\dot{u}} \quad \text { for } \quad x_{s, r}-e_{s} \in \mathcal{X}_{i}, i \in \mathcal{I} .
$$

Next, the time derivative of the control input $\underline{u}$ is replaced by the control input in error-space [9], which is defined as

$$
\underline{\mu}=\underline{\dot{u}} \text {. }
$$


When we define the state vector of the error dynamics as

$$
\underline{q}=\left[\begin{array}{ll}
e_{s} & \dot{e}_{s}
\end{array}\right]^{T},
$$

we can write the flow conditions in error space in standard state-variable form:

$$
\underline{\dot{q}}=F \underline{q}+G_{i} \underline{\mu} \quad \text { for } \quad\left(x_{s, r}-\left[\begin{array}{ll}
1 & 0
\end{array}\right] \underline{q}\right) \in \mathcal{X}_{i}, i \in \mathcal{I} .
$$

In this notation, the system matrix is defined as $F=$ $\left[\begin{array}{ll}0 & 1 \\ 0 & 0\end{array}\right]$, whereas the input matrix is defined as $G_{i}=$ $\left[\begin{array}{ll}\underline{0} & -B_{i}^{T}\end{array}\right]^{T}$.

Regarding the jump conditions, the physical interpretation of the system at hand shows that switching from regime $k$ to regime $k+1$ and vice versa is possible, with $k \in \mathcal{K}$, $\mathcal{K}=\{1,2\} .{ }^{1}$ When we consider the transition from regime $k$ to regime $k+1$, the following relation holds for the sheet tracking error at the switching boundary:

$$
e_{s}^{+}\left(t_{s}\right)=e_{s}^{-}\left(t_{s}\right)
$$

with $e_{s}^{+}\left(t_{s}\right):=\lim _{t \downarrow t_{s}} e_{s}(t)$ and $e_{s}^{-}\left(t_{s}\right):=\lim _{t \uparrow t_{s}} e_{s}(t)$, where $t_{s}$ is the switching time. From (7) it can be seen that $e_{s}$ is continuous at the switching boundary, which is supported by the physics of the system, as the sheet cannot make instantaneous jumps in its position. The jump conditions for $\dot{e}_{s}$ are derived from (2). For $\dot{e}_{s}^{+}\left(t_{s}\right)$, the following relation holds:

$$
\dot{e}_{s}^{+}\left(t_{s}\right)=\dot{x}_{s, r}\left(t_{s}\right)-B_{k+1} \underline{u}\left(t_{s}\right), k \in \mathcal{K},
$$

whereas for $\dot{e}_{s}^{-}\left(t_{s}\right)$ it holds that

$$
\dot{e}_{s}^{-}\left(t_{s}\right)=\dot{x}_{s, r}\left(t_{s}\right)-B_{k} \underline{u}\left(t_{s}\right), k \in \mathcal{K} \text {. }
$$

Subtraction of (9) from (8) yields the desired jump condition for $\dot{e}_{s}$ :

$$
\dot{e}_{s}^{+}\left(t_{s}\right)=\dot{e}_{s}^{-}\left(t_{s}\right)+\left(B_{k}-B_{k+1}\right) \underline{u}\left(t_{s}\right), k \in \mathcal{K} .
$$

Given (7) and (10), the jump conditions can be represented as

$\underline{q}^{+}\left(t_{s}\right)=\left[\begin{array}{ll}1 & 0 \\ 0 & 1\end{array}\right] \underline{q}^{-}\left(t_{s}\right)+\left[\begin{array}{c}\underline{0}^{T} \\ B_{k}-B_{k+1}\end{array}\right] \underline{u}\left(t_{s}\right), k \in \mathcal{K}$,

with $\underline{q}^{-}\left(t_{s}\right):=\left[\begin{array}{ll}e_{s}^{-}\left(t_{s}\right) & \dot{e}_{s}^{-}\left(t_{s}\right)\end{array}\right]^{T}$ and $\underline{q}^{+}\left(t_{s}\right):=$ $\left[\begin{array}{ll}e_{s}^{+}\left(t_{s}\right) & \dot{e}_{s}^{+}\left(t_{s}\right)\end{array}\right]^{T}$. Hence, the complete model of the open-loop sheet dynamics in error space is given by the flow conditions (6) and the jump conditions (11). This means that applying the well-known error space approach to a PWL system yields an error dynamics that is PWL and has resets.

\footnotetext{
${ }^{1}$ Note that the index $k$ is used to indicate the regimes between which a transition is made, whereas the index $i$ is used to indicate the regimes in all other cases.
}

\section{CONTROLLER SYNTHESIS}

For controlling the piecewise linear flow dynamics in error space (6) in combination with the jump conditions (11), we propose a control law that is based on state feedback of the error dynamics:

$$
\underline{\mu}=-K \underline{q},
$$

with $K$ the matrix with feedback gains to be calculated. Substitution of (12) into (6) yields the closed-loop flow dynamics in error space:

$$
\underline{\dot{q}}=\left(F-G_{i} K\right) \underline{q} \quad \text { for } \quad\left(x_{s, r}-\left[\begin{array}{ll}
1 & 0
\end{array}\right] \underline{q}\right) \in \mathcal{X}_{i}, i \in \mathcal{I} .
$$

For the derivation of the closed-loop jump conditions, first $\underline{u}$ is derived by substitution of (12) into (4) and integration of the resulting equation. Hence, for each $i$-th element of $\underline{u}$, $u_{i}$, the following relation holds:

$$
u_{i}(t)=-K(i, 1) \int_{t_{0}}^{t} e_{s}(\tau) \mathrm{d} \tau-K(i, 2) e_{s}(t), i \in \mathcal{I}
$$

with $t_{0}$ the initial time, $u_{i}\left(t_{0}\right)=0$, and $K(i, j)$ the $j$ th element of the $i$-th row of $K, j \in\{1,2\}$. As can be seen from (14), the control law for each region consists of a proportional-integral (PI) controller.

The second step in deriving the closed-loop jump conditions is substitution of (14) into (10), yielding the closed-loop jump condition for $\dot{e}_{s}$ when considering the transition from regime $k$ to regime $k+1$ :

$$
\begin{aligned}
\dot{e}_{s}^{+}\left(t_{s}\right)= & \dot{e}_{s}^{-}\left(t_{s}\right)-B_{k}(k) K(k, 1) \int_{t_{0}}^{t_{s}} e_{s}(\tau) \mathrm{d} \tau- \\
& -B_{k}(k) K(k, 2) e_{s}\left(t_{s}\right)+ \\
& +B_{k+1}(k+1) K(k+1,1) \int_{t_{0}}^{t_{s}} e_{s}(\tau) \mathrm{d} \tau+ \\
& +B_{k+1}(k+1) K(k+1,2) e_{s}\left(t_{s}\right), k \in \mathcal{K} .
\end{aligned}
$$

In (15), $B_{k}(k)$ represents the $k$-th element of $B_{k}$, i.e. its only nonzero element. Given (7) and (15), the closed-loop jump conditions can be represented as

$$
\begin{aligned}
\underline{q}^{+}\left(t_{s}\right)= & {\left[\begin{array}{cc}
1 & 0 \\
B_{k+1}(k+1) K(k+1,2)- & 1 \\
-B_{k}(k) K(k, 2) & 1
\end{array}\right] \underline{q}^{-}\left(t_{s}\right)+} \\
0 & {\left[\begin{array}{c}
B_{k+1}(k+1) K(k+1,1)- \\
-B_{k}(k) K(k, 1)
\end{array}\right] \int_{t_{0}}^{t_{s}} e_{s}(\tau) \mathrm{d} \tau } \\
& +\left[\begin{array}{c}
0 \\
\left.B_{k}\right)
\end{array}\right. \\
& +\left[\begin{array}{c}
B_{k+1}(k+1) K(k+1,1)- \\
-B_{k}(k) K(k, 1)
\end{array}\right] \int_{t_{0}}^{t_{s}} e_{s}(\tau) \mathrm{d} \tau, \\
& {\left[\begin{array}{c}
k \in \mathcal{K} .
\end{array}\right.}
\end{aligned}
$$

To stabilize the error dynamics in $q=\underline{0}$, which implies that $e_{s}=0$ and $\dot{e}_{s}=0$, we require $q=\underline{0}$ to be an equilibrium of the closed-loop system (1) $\overline{3})-(16)$. The only requirement for $\underline{q}=\underline{0}$ to be an equilibrium is that the second term of (16) is equal to $\underline{0}$. Clearly, a sufficient condition for this to realize is $B_{k+1}(k+1) K(k+1,1)=B_{k}(k) K(k, 1)$ via the choice of $K(k, 1)$ and $K(k+1,1)$. If the integral term in (16) is nonzero, which is to be expected in practical cases, 
the above mentioned condition is also a necessary one. In other words, the feedback controllers (14) have to be at least partially linearizing to enforce the origin to be a globally asymptotically stable equilibrium in the sense of Lyapunov. Alternatively, if one requires both $B_{k+1}(k+1) K(k+1,2)=$ $B_{k}(k) K(k, 2)$ and $B_{k+1}(k+1) K(k+1,1)=B_{k}(k) K(k, 1)$ to hold via the choice of $K$, applying the resulting controller to the sheet flow model (1) yields a linear closed-loop system. From this we can conclude that in this case the controller linearizes the sheet flow dynamics. In summary, two types of controllers are considered:

- Fully linearizing controllers. To obtain this type of controllers, the gains should be chosen such that both $B_{k+1}(k+1) K(k+1,2)=B_{k}(k) K(k, 2)$ and $B_{k+1}(k+$ $1) K(k+1,1)=B_{k}(k) K(k, 1)$. Given this type of controllers, $q=\underline{0}$ is an equilibrium point of the closedloop system and jumps in the error states will not occur.

- Partially linearizing controllers. To obtain this type of controllers, the gains should be chosen such that $B_{k+1}(k+1) K(k+1,1)=B_{k}(k) K(k, 1)$. Given this type of controllers, $q=\underline{0}$ is an equilibrium point of the closed-loop system and jumps in $\dot{e}_{s}$ will not be eliminated.

Given a partially linearizing feedback controller, the expression for the closed-loop jump conditions becomes:

$$
\underline{q}^{+}=R_{k, k+1} \underline{q}^{-}, k \in \mathcal{K} .
$$

In analogy with the derivation of (17), the closed-loop jump conditions can also be derived for a transition from regime $k+1$ to regime $k$, i.e. when the system switches back to the previous regime, yielding:

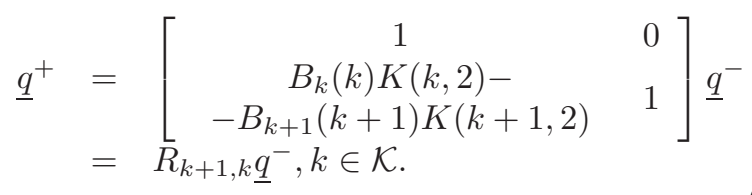

Given (16) and (18), the closed-loop jump conditions that hold when switching from regime $k$ to regime $k+1$ and back are related via

$$
R_{k, k+1}=R_{k+1, k}^{-1}, k \in \mathcal{K} .
$$

Focussing on the transition from region $k$ to region $k+$ 1 , the stability analysis of the closed-loop error dynamics (13), (17) encompasses two parts: the stability analysis of the flow dynamics in each regime and the stability analysis at the switching boundary connecting two regimes. By making use of the work presented in [1], [13], it can be analyzed whether the closed-loop flow dynamics in error space in (13) are Globally Exponentially Stable (GES). By considering exponential stability, the rate of convergence of the tracking error to zero can be adjusted such that prespecified performance properties are satisfied. For the stability analysis, a piecewise quadratic Lyapunov function candidate is proposed:

$$
V_{i}(\underline{q})=\underline{q}^{T} P_{i} \underline{q}, i \in \mathcal{I},
$$

with

$$
P_{i}=P_{i}^{T} \succ 0, i \in \mathcal{I} \text {. }
$$

To prove that the flow dynamics are GES, the following set of matrix inequalities in $P_{i}$ and $K$ must hold:

$$
\begin{aligned}
0 & \succ \dot{V}_{i}(\underline{q})+\alpha V_{i}(\underline{q})= \\
& =\left(F-G_{i} K\right)^{T} P_{i}+P_{i}\left(F-G_{i} K\right)+\alpha P_{i}, i \in \mathcal{I},
\end{aligned}
$$

with $\alpha>0$ representing the decay rate of the Lyapunov function. This parameter is chosen a priori in relation to the transient behavior of the error dynamics, and therefore in relation to the desired tracking performance. From (22), it becomes clear that both the stability analysis of the flow dynamics and the calculation of the controller gains $K$ can be carried out by solving a single set of matrix inequalities. In other words, the free parameters to be solved, i.e. the Lyapunov matrices $P_{i}$ and the matrix with controller gains $K$, depend on each other.

This dependency is also present in the stability analysis at the switching boundary connecting two regimes. To characterize the dependency, we consider both the transition from regime $k$ to regime $k+1$ and vice versa. In both transitions, no extra energy is allowed to enter the system, i.e. the Lyapunov function active in a new regime must be smaller than or equal to the Lyapunov function active in the previous regime. Keeping this in mind, we start with the transition from regime $k$ to regime $k+1$, and we require:

$$
\left(\underline{q}^{+}\right)^{T} P_{k+1} \underline{q}^{+} \leq\left(\underline{q}^{-}\right)^{T} P_{k} \underline{q}^{-}, k \in \mathcal{K} .
$$

To show the relation between $P_{i}$ and the controller parameters, two inequalities describing the relation between $P_{k}$, $P_{k+1}, R_{k, k+1}$, and $R_{k+1, k}$ can be derived from (23) by making use of the closed-loop jump conditions (17). First of all, (17) is substituted into (23) to obtain a relation between $P_{k}, P_{k+1}$, and $R_{k, k+1}$ :

$$
\left(\underline{q}^{-}\right)^{T} R_{k, k+1}^{T} P_{k+1} R_{k, k+1} \underline{q}^{-} \leq\left(\underline{q}^{-}\right)^{T} P_{k} \underline{q}^{-}, k \in \mathcal{K} .
$$

In the derivation of the second inequality, first an expression for $\underline{q}^{-}$is derived from (17) and (19):

$$
\begin{aligned}
\underline{q}^{-} & =R_{k, k+1}^{-1} \underline{q}^{+} \\
& =R_{k+1, k} \underline{q}^{+}, k \in \mathcal{K} .
\end{aligned}
$$

Substitution of (25) into (23) yields the desired second inequality, which describes the relation between $P_{k+1}, P_{k}$, and $R_{k+1, k}$ :

$$
\left(\underline{q}^{+}\right)^{T} P_{k+1} \underline{q}^{+} \leq\left(\underline{q}^{+}\right)^{T} R_{k+1, k}^{T} P_{k} R_{k+1, k} \underline{q}^{+}, k \in \mathcal{K} .
$$

In finding a relation between $P_{i}$ and the controller parameters, the second transition we consider is the one from regime $k+1$ to regime $k$. In analogy with the derivation of (24) and (26), two additional relations between $P_{k}, P_{k+1}, R_{k, k+1}$, and $R_{k+1, k}$ can be obtained:

$$
\begin{aligned}
& \left(\underline{q}^{-}\right)^{T} R_{k+1, k}^{T} P_{k} R_{k+1, k} \underline{q}^{-} \leq\left(\underline{q}^{-}\right)^{T} P_{k+1} \underline{q}^{-}, k \in \mathcal{K}, \\
& \left(\underline{q}^{+}\right)^{T} P_{k} \underline{q}^{+} \leq\left(\underline{q}^{+}\right)^{T} R_{k, k+1}^{T} P_{k+1} R_{k, k+1} \underline{q}^{+}, k \in \mathcal{K} .
\end{aligned}
$$


From the combination of (24) and (28), which hold for all $\underline{q}^{-}$and $\underline{q}^{+}$, we know that

$$
P_{k}=R_{k, k+1}^{T} P_{k+1} R_{k, k+1}, k \in \mathcal{K},
$$

whereas the combination of (26) and (27), which also hold for all $\underline{q}^{-}$and $\underline{q}^{+}$, results in

$$
P_{k+1}=R_{k+1, k}^{T} P_{k} R_{k+1, k}, k \in \mathcal{K} .
$$

Note that (29) can be rewritten into (30) and vice versa via (19).

With the derivation of (29) and (30) we have obtained the desired relations between $P_{i}$ and the controller parameters. From these relations, a number of interesting observations can be made. First of all, if the controller structure is chosen such that a linear closed-loop system is obtained, there will be no jumps in $e_{s}$ and $\dot{e}_{s}$, independent of their values at the switching boundary. For that case $R_{k, k+1}=R_{k+1, k}=I$ holds and it can be observed that $P_{k}=P_{k+1}$ in (29) and (30), and hence, a common quadratic Lyapunov function is obtained, which yields the stability of the closed-loop system under arbitrary switching. This reasoning can also be carried out in the opposite direction. Suppose a common quadratic Lyapunov function is used to prove the stability of the closed-loop system under arbitrary switching. According to (29), the following holds for $P$ in that case:

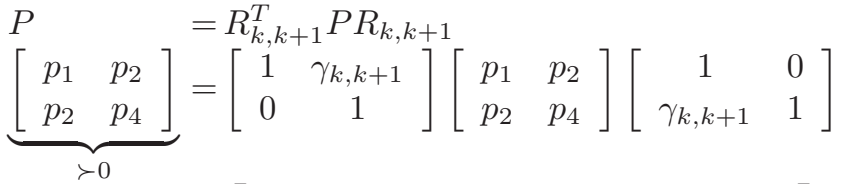

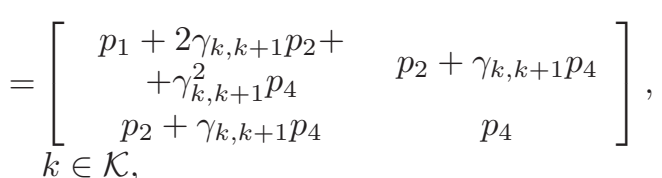

with $\gamma_{k, k+1}=B_{k+1}(k+1) K(k+1,2)-B_{k}(k) K(k, 2)$. Since the symmetric matrix $P$ has to be positive definite, we know from Sylvester's theorem [14] that the following necessary and sufficient conditions have to hold:

$$
\begin{gathered}
p_{1}+2 \gamma_{k, k+1} p_{2}+\gamma_{k, k+1}^{2} p_{4}>0, k \in \mathcal{K}, \\
\operatorname{det}(P)>0, k \in \mathcal{K} .
\end{gathered}
$$

If we now suppose that $\gamma_{k, k+1} \neq 0$, then both $p_{2}$ and $p_{4}$ have to be equal to zero to fulfill (31). However, in that case (33) cannot hold anymore. Therefore, $\gamma_{k, k+1}$ has to be equal to 0 , which implies that $B_{k+1}(k+1) K(k+1,2)=$ $B_{k}(k) K(k, 2)$ and, hence, $R_{k, k+1}=I$. Hence, suppose there exists a partially linearizing controller $K$ for controlling the sheet dynamics in error space under consideration, and there exists a piecewise quadratic Lyapunov function. Then the controller results in a linear closed-loop system if and only if the Lyapunov function is a common one. ${ }^{2}$

\footnotetext{
${ }^{2}$ Note that in case of piecewise smooth Lyapunov functions, the equivalent of (29) can also be derived, yielding $V_{k}(\underline{q})=V_{k+1}\left(R_{k, k+1} \underline{q}\right)$. Also in this case it holds that if $R_{k, k+1}=I$, a common Lyapunov function will be obtained.
}

\section{A. Linearizing Controller Synthesis}

When it is desired to use a sheet feedback controller that completely linearizes the system, the jump conditions can be left out of consideration since $\underline{q}^{+}=\underline{q}^{-}$in (17)-(18). From Section III we know that when all transitions from regime $k$ to regime $k+1$ and vice versa are allowed, a linearizing feedback controller will be obtained if $R_{k, k+1}=R_{k+1, k}=I$, as a result of which a common quadratic Lyapunov function can be used for the stability analysis, i.e. $P_{i}=P$ in (21)-(22). To calculate the controller gains, the constraints $R_{k, k+1}=$ $R_{k+1, k}=I$ can be added to the matrix inequalities (21)-(22). Alternatively, matrix inequalities analogous to (21)-(22) can be derived for one regime, e.g. for the first one, yielding

$$
\begin{gathered}
P=P^{T} \succ 0, \\
0=\left(F-\tilde{G}_{1} K(1, \bullet)\right)^{T} P+P\left(F-\tilde{G}_{1} K(1, \bullet)\right)+\alpha P,
\end{gathered}
$$

with $\tilde{G}_{1}=\left[\begin{array}{ll}0 & -B_{1}(1)\end{array}\right]^{T}$ and with $K(1, \bullet)$ the first row of $K$ containing the controller gains for the first subsystem. After solving these matrix inequalities, the controller gains for the other regimes can be obtained via scaling. However, since these matrix inequalities are nonlinear in the unknown matrices $P$ and $K(1, \bullet)$, a linearizing change of variable variables is applied [13]. More specifically, we pre- and postmultiply (34) and (35) with $P^{-1}$ and substitute $X=P^{-1}$ and $Y=K(1, \bullet) P^{-1}$ to obtain the following set of LMIs in the free variables $X$ and $Y$ :

$$
\begin{gathered}
0 \prec X=X^{T}, \\
0 \succ F X+X F^{T}-\tilde{G}_{1} Y-Y^{T} \tilde{G}_{1}^{T}+\alpha X .
\end{gathered}
$$

The calculation of the free variables amounts to solving a feasibility problem for which efficient software is available [15]. After solving the LMIs (36)-(37), the controller gains can be calculated using

$$
K(1, \bullet)=Y X^{-1}
$$

Next, $K(2, \bullet)$ and $K(3, \bullet)$ can be derived via

$$
K(2, \bullet)=s_{2} K(1, \bullet),
$$

and

$$
K(3, \bullet)=s_{3} K(1, \bullet),
$$

with $s_{2}:=\frac{B_{1}(1)}{B_{2}(2)}$ and $s_{3}:=\frac{B_{1}(1)}{B_{3}(3)}$ the scaling factor for the second and third subsystem, respectively.

\section{B. Partially linearizing Controller Synthesis}

To exploit the possibilities of using a feedback controller that does not completely linearize the system, resulting in more freedom in the choice of the controller parameters, this section presents an approach for calculating these parameters. For simplicity, we use a rather ad-hoc approach. Further research should be carried out to investigate if the calculation of the controller parameters and the Lyapunov functions can be preformed via efficient techniques, e.g. using LMIs [13]. 
Focussing first on subsystem 1, the controller parameters for this subsystem can be calculated by solving (36)-(37). For the calculation of the controller parameters for the second subsystem, i.e. $K(2, \bullet)$, the following approach can be used:

1) To ensure that $q=\underline{0}$ is an equilibrium of our system, the equality $B_{2}(2) K(2,1)=B_{1}(1) K(1,1)$ has to be enforced, as recognized in Section III. Hence, $K(2,1)$ can be calculated using

$$
K(2,1)=s_{2} K(1,1) .
$$

2) Given $K(2,1), K(2,2)$ remains the only unknown controller parameter for the second subsystem, as the Lyapunov matrix $P_{2}$ can be expressed as a function of this parameter via (30). Given this matrix, both (21) and (22) are evaluated for $i=2$, given a range of values of $K(2,2)$. This range can, for example, be chosen in the neighborhood of the value for $K(2,2)$ that linearizes the system built up from the first and second subsystem, i.e. $K(2,2)=s_{2} K(1,2)$.

3 ) From the values of $K(2,2)$ for which (21) and (22) are satisfied, one can be chosen that does not linearize the system, i.e. for which $K(2,2)=s_{2} K(1,2)$ does not hold. The actual choice of $K(2,2)$ depends on the requirements, e.g. the minimization of a performance index containing the control input or the enforcement of different control bandwidths in the various subsystems. With the choice of $K(2,2)$, the partially linearizing controller for the second subsystem has been found.

The calculation of the controller parameters for the third subsystem can be carried out in analogy with steps 1-3 described above.

\section{EXPERIMENTAL SETUP}

To validate the proposed control design, the experimental printer paper path setup shown in Fig. 3 is used. It consists of a paper input module and a paper path with five pinches. In the experiments the second, third and fourth pinch are the pinches where the actual control action takes place. In the remainder of this paper we will refer to these pinches as pinch $\mathrm{P} 1$, pinch $\mathrm{P} 2$, and pinch $\mathrm{P} 3$ respectively. Each pinch is connected to a motor via a gear belt. The transmission ratios are $n_{1}=\frac{18}{37}, n_{2}=\frac{16}{30}$, and $n_{3}=\frac{18}{37}$. The radii $r_{P i}$ of the pinches are equal to $14 \cdot 10^{-3} \mathrm{~m}$. The angular positions of the motors are measured via optical incremental encoders with

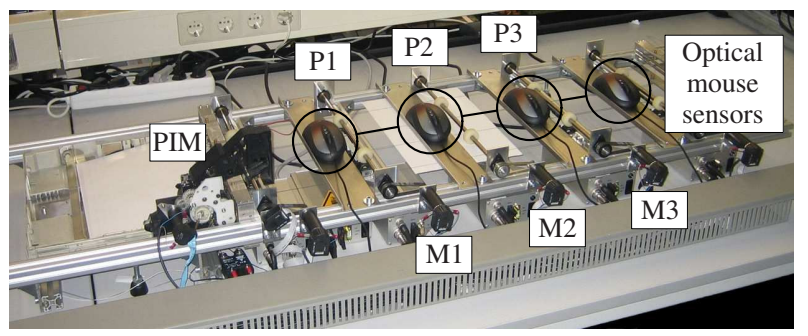

Fig. 3. Photo of the experimental paper path setup. a resolution of 2000 increments per revolution. The motors are $10 \mathrm{~W}$ DC motors driven by current amplifiers. Both the amplifiers and the encoders are connected to a PC-based control system. This system consists of three TUeDACS USB I/O devices [16], a Pentium 4 host computer running RTAI/Fusion Linux and Matlab/Simulink. The sheets are guided through the paper path using thin steel wires and their position is measured using optical mouse sensors with a resolution of approximately $10 \cdot 10^{-6} \mathrm{~m}$, which are directly connected to the host computer via USB.

\section{RESUlTS}

Given the approaches presented in Subsections III-A and III-B, together with the system parameters given in Section IV, the controller gains are calculated to be

$$
K_{l}=\left[\begin{array}{ll}
-2.3 \cdot 10^{5} & -9.9 \cdot 10^{3} \\
-2.4 \cdot 10^{5} & -1.0 \cdot 10^{4} \\
-2.2 \cdot 10^{5} & -9.6 \cdot 10^{3}
\end{array}\right]
$$

and

$$
K_{p l}=\left[\begin{array}{ll}
-2.3 \cdot 10^{5} & -8.6 \cdot 10^{3} \\
-2.3 \cdot 10^{5} & -9.5 \cdot 10^{3} \\
-2.2 \cdot 10^{5} & -8.9 \cdot 10^{3}
\end{array}\right]
$$

for the linearizing and partly linearizing controllers, respectively. From these gains it can be concluded that the gains in (42) indeed linearize the system, whereas the gains in (43) result in partial linearization. Given the calculated controller gains, experiments have been carried out to validate the control design in practice. For the sheet motion task, a constant velocity of $0.3 \mathrm{~ms}^{-1}$ is chosen that had to be tracked throughout the paper path. The results of the experiments can be seen in Fig. 4. The experimentally obtained sheet tracking error using the fully linearizing sheet feedback controller is depicted in Fig. 4(a), whereas the tracking error obtained using the partially linearizing controller is shown in Fig. 4(b). Both figures also show the results obtained in simulation. It can be seen that the responses in both figures resemble each other strongly. Hence, for this setup the benefit of using partially linearizing controllers instead of fully linearizing ones is not clear. However, the question remains if control problems exist, e.g. $H_{\infty}$ control problems, in which partially linearizing controllers can have benefits of linearizing ones. The main difference between the two tracking errors shown in Fig. 4 is the maximum deviation from the desired zero error level after entering a new pinch, caused by a small difference in bandwidth: approximately $11 \mathrm{~Hz}$ and $10 \mathrm{~Hz}$ for the fully linearizing and partially linearizing controller, respectively. As in the simulation, the large transient behavior just after the moment the sheet enters the paper path is caused by the difference between the sheet reference velocity and the initial actual sheet velocity. Moreover, the increase in tracking error when the sheet enters the regimes two and three is caused by the deviation of the implemented transmission ratios with respect to ones used in the calculation of the controller gains. Also these increases are controlled towards zero quickly. From this result, it can be concluded that robustness against parameter uncertainties 


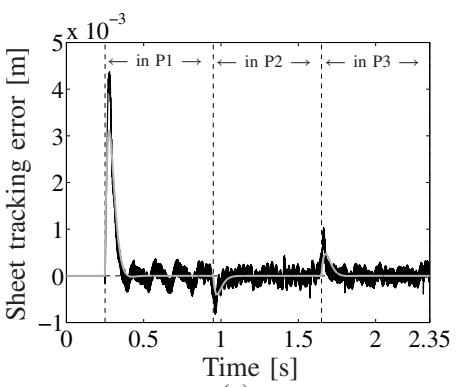

(a)

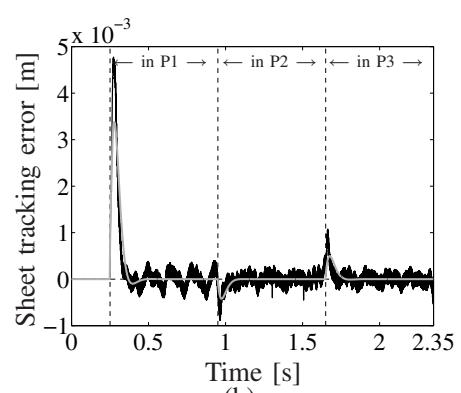

(b)
Fig. 4. Sheet tracking error obtained in experiments (black) and simulation (gray) using the linearizing (a) and the partially linearizing (b) sheet feedback controllers.

has been obtained, although these uncertainties were not taken into account in the controller synthesis.

\section{CONCLUSIONS AND RECOMMENDATIONS}

In this paper, tracking control for piecewise linear systems using an error space approach has been considered. The PWL model of the sheet flow in a printer paper path has been written in terms of its error dynamics. A distinguishing feature with respect to the derivation of the error dynamics of linear systems was the introduction of jump conditions describing the system behavior at the switching boundary. Hence, for the original PWL model without jump relations, a model of the error dynamics has been obtained that is piecewise linear and has jumps. To make the origin an equilibrium of the error dynamics, some linearizing restrictions had to be enforced on the controllers. Based on this observation, two types of controllers have been designed, yielding either a linear or a PWL, discontinuous closed-loop system. Stability analysis of both systems has yielded much insight in the relation between the type of controller used and the type of Lyapunov function needed to prove the stability of this type of systems. Experimental results have shown that both types of controllers result in stability of the closed-loop system. Moreover, it has been observed that some inherent robustness against uncertainties in the model parameters has been obtained.

In general, this work indicates that an error space approach for PWL systems might not be so fruitful as for linear systems due to the complicated error dynamics that arise. Therefore, we have also considered other approaches to tracking control, based on $H_{\infty}$ design techniques [8]. However, it is still of interest to see whether or not partially linearizing controllers can have benefits of linearizing ones. Currently it is under investigation if this is the case for certain $H_{\infty}$ control problems.

\section{REFERENCES}

[1] R. A. DeCarlo, M. S. Branicky, S. Pettersson, and B. Lennartson, "Perspectives and results on the stability and stabilizability of hybrid systems," Proc. IEEE, vol. 88(7), pp. 1069-1082, July 2000.

[2] M. Johansson and A. Rantzer, "Computation of piecewise quadratic lyapunov functions for hybrid systems," IEEE Trans. Automat. Contr., vol. 43(4), pp. 555-559, April 1998.

[3] A. Rantzer and M. Johansson, "Piecewise linear quadratic optimal control," IEEE Trans. Automat. Contr., vol. 45(4), pp. 629-637, April 2000.

[4] A. Hassibi and S. Boyd, "Quadratic stabilization and control of piecewise-linear systems," in Proc. of the American Control Conference, Philadelphia, PA, USA, June 1998, pp. 3659-3664.

[5] L. Rodrigues, A. Hassibi, and J. P. How, "Output feedback controller synthesis for piecewise-affine system with multiple equilibria," in Proc. of the American Control Conference, Chicago, Illinois, USA, June 2000, pp. 1784-1789.

[6] S. Solyom and A. Rantzer, "The servo problem for piecewise linear systems," in Proc. of the Fifteenth International Symposium on Mathematical Theory of Networks and Systems, Notre Dame, IN, August 2002.

[7] G. Feng, G. P. Lu, and S. S. Zhou, "An approach to $H_{\infty}$ controller synthesis of piecewise linear systems," Communications in Information and Systems, vol. 2(3), pp. 245-254, December 2002.

[8] B. H. M. Bukkems, Sheet Feedback Control Design in a Printer Paper Path, Ph.D. thesis, Eindhoven University of Technology, Eindhoven, The Netherlands, 2007.

[9] G. F. Franklin, J. D. Powell, and A. Emami-Naeini, Feedback control of dynamic systems. Upper Saddle River, New Jersey, USA: Prentice Hall, 2002.

[10] M. Kruciński, Feedback Control of Photocopying Machinery, Ph.D. thesis, University of California Berkeley, CA, USA, 2000.

[11] C. Cloet, A Mechatronics Approach to Copier Paperpath Design, Ph.D. thesis, University of California Berkeley, CA, USA, 2001.

[12] E. D. Sontag, "Nonlinear regulation: The piecewise linear approach," IEEE Trans. Automat. Contr., vol. 26(2), pp. 346-381, April 1981.

[13] S. Boyd, L. El Ghaoui, E. Feron, and V. Balakrishnan, Linear Matrix Inequalities in System and Control Theory. Philadelphia, PA, USA: Volume 15 of SIAM Studies in Applied Mathematics, June 1994.

[14] J.-J. E. Slotine and W. Li, Applied Nonlinear Control. Englewood Cliffs, New Jersey, USA: Prentice Hall, 1991.

[15] P. Gahinet, A. Nemirovski, A. J. Laub, and M. Chilali, LMI Control Toolbox for Use with Matlab. Natick, MA, USA: The Mathworks Inc., May 1995.

[16] M. J. G. van de Molengraft, A. de Kraker, and M. Steinbuch, "Integrating experimentation into control courses," IEEE Control Syst. Mag., vol. 25(1), pp. 40-44, February 2005. 